\title{
Parvovirus B19 infection associated with hemolytic anemia and cranial polyneuropathy
}

\author{
João Sequeira $^{1}\left(\mathbb{D} \cdot\right.$ Ana Calado $^{1} \cdot$ Margarida Dias $^{1} \cdot$ Manuel Manita $^{1}$
}

Received: 15 May 2017 / Revised: 29 June 2017 / Accepted: 26 July 2017

(C) Journal of NeuroVirology, Inc. 2017

\begin{abstract}
Parvovirus B19 (PB19) is a common, widespread, small, single-stranded DNA virus which has been linked with a broad spectrum of clinical illnesses, including a variety of neurological complications such as encephalitis, meningitis, myelitis, stroke, cerebellar ataxia, and neuropathy. The authors describe a case of PB19 infection associated with hemolytic anemia and cranial polyneuropathy involving the second and third cranial nerves in a 23-year-old immunocompetent woman. The diagnosis of acute PB19 infection was established with detection of positive DNA and anti-PB19 IgM antibodies in blood samples. Antiganglioside antibody studies were performed and serum anti-GD1b IgG was strongly positive. Further investigation was normal or negative, excluding other infectious or autoimmune disorders. The patient was initially treated with a 5-day course of intravenous immunoglobulin (IGIV). Because of incomplete neurological recovery, methylprednisolone was also administered 7 days after IGIV therapy initiation. Complete resolution of clinical symptoms was observed 3 months after disease onset at follow-up visit, despite the persistence of PB19 DNA and anti-PB19 IgM antibodies in serum 5 months after the initial presentation. Our report provides evidence that PB19 could affect both the central and peripheral nervous system, possibly by triggering an autoimmune mechanism that leads to autoantibody production.
\end{abstract}

Keywords Parvovirus B19 · Infection · Autoantibodies · Hemolytic anemia $\cdot$ Cranial neuropathy

João Sequeira

joaopfsequeira@gmail.com

1 Department of Neurology, Centro Hospitalar de Lisboa Central, E.P.E. - Hospital de Santo António dos Capuchos, Alameda Santo António dos Capuchos, 1169-050 Lisbon, Portugal

\section{Introduction}

Parvovirus B19 (PB19) is a common, widespread, small, single-stranded DNA virus which has been linked with a broad spectrum of clinical illnesses, ranging from selflimiting erythema infectiosum in the healthy host to lifethreatening complications in immunocompromised patients (Qiu et al. 2017; Broliden et al. 2006). In rare cases, PB19 infection could be related with less commonly recognized and infrequently reported neurological manifestations. We describe a case of PB19 infection associated with cranial polyneuropathy and hemolytic anemia in a 23 -year-old immunocompetent woman.

\section{Case report}

A previously healthy 23-year-old woman presented to our emergency department with a 2-day history of acute right eye pain during eye movement followed by ipsilateral blurring vision, pupillary asymmetry, and double vision 2 weeks after a spontaneously resolved diarrheal episode. Neurological examination showed an uncorrected visual acuity of $7 / 10$ in her right eye and a relative afferent pupillary defect, anisocoria with an ipsilateral dilated pupil, paresis of right eye adduction, and vertical diplopia in all directions of gaze. The remaining clinical examination was otherwise normal. Cerebral computed tomography, brain magnetic resonance imaging (Fig. 1), and magnetic resonance angiography were normal. Initial laboratory testing revealed a mild increase of total bilirubin $(1.85 \mathrm{mg} / \mathrm{dL}$; normal range $<1.20 \mathrm{mg} / \mathrm{dL})$ and direct bilirubin $(0.52 \mathrm{mg} / \mathrm{dL}$; normal range $<0.50 \mathrm{mg} / \mathrm{dL}$ ) levels, whereas other liver and renal function tests, hemoglobin, white blood cell count, erythrocyte sedimentation rate, and C-reactive protein were normal. Autoimmune screening was negative for antinuclear antibodies, 


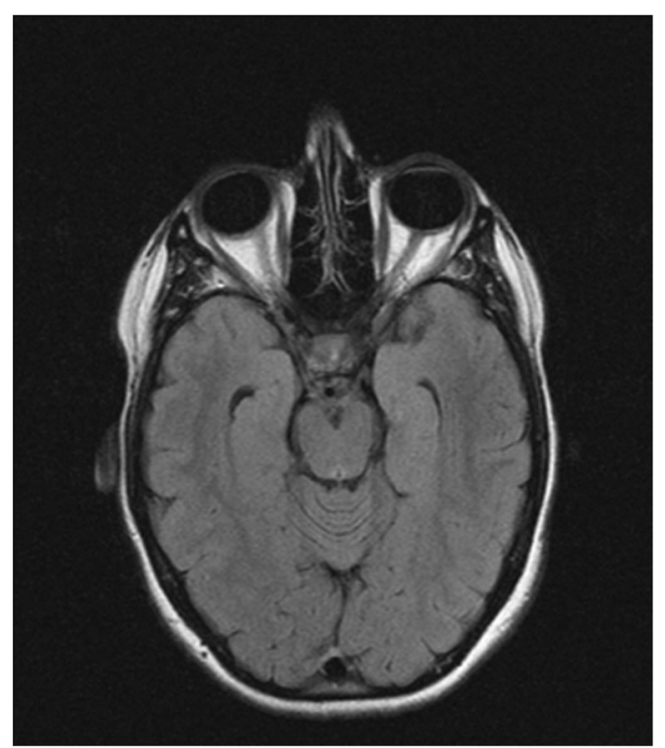

Fig. 1 Normal brain magnetic resonance imaging (axial T2 FLAIR)

antineutrophil cytoplasmic antibodies, antithyroid antibodies, and serum angiotensin converting enzyme. Antiganglioside antibody studies were performed and serum anti-GD1b IgG was strongly positive. Cerebrospinal fluid (CSF) exhibited normal cell count and protein level, absence of IgG bands or intrathecal IgG synthesis, and the presence of a damaged blood-brain barrier. Serological tests in serum and CSF excluded active infection due to enterovirus, herpes simplex virus 1 and 2, varicella zoster virus, cytomegalovirus, Epstein-Barr virus, hepatitis B virus, hepatitis $C$ virus, human immunodeficiency virus 1 and 2, Treponema pallidum, and Borrelia burgdorferi. Additional blood investigations displayed positive anti-PB19 IgM (3.20; positive $\geq 1.0)$ and $\operatorname{IgG}(40.90$; positive $\geq 1.0)$ antibodies, and PB19 DNA was detected using polymerase chain reaction
(PCR). Search for PB19 DNA in CSF with PCR was negative. A diagnosis of PB19-associated cranial polyneuropathy involving the second and third cranial nerves was made and the patient was initially treated with a 5-day course of intravenous immunoglobulin (IVIG) $30 \mathrm{~g} /$ day. Because of incomplete neurological recovery, methylprednisolone $1 \mathrm{~g}$ daily for 5 days was also administered 7 days after IGIV therapy initiation. During her stay in the hospital, she developed an episode of self-limited hemolytic anemia with a low hemoglobin count $(9.7 \mathrm{~g} /$ $\mathrm{dL}$; normal range $>12.0 \mathrm{~g} / \mathrm{dL}$ ) and increased total bilirubin $(5.72 \mathrm{mg} / \mathrm{dL})$ and direct bilirubin $(0.66 \mathrm{mg} / \mathrm{dL})$ levels. Progressive neurological improvement was observed and the patient was discharged 21 days after admittance, maintaining anisocoria and paresis of right eye adduction. Complete resolution of clinical symptoms was observed 3 months after admission at follow-up visit. Repeated laboratory workup in serum 5 months after the initial presentation evidenced the persistence of PB19 DNA and anti-PB19 $\operatorname{IgM}(1.10$; positive $\geq 1.0)$ and $\operatorname{IgG}(32.50$; positive $\geq 1.0$ ) antibodies but was negative regarding antiganglioside antibody studies.

\section{Discussion}

A variety of neurological complications have been associated with PB19 infection, including encephalitis, meningitis, myelitis, stroke, cerebellar ataxia, and neuropathy (Barah et al. 2003; Barah et al. 2014; Douvoyiannis et al. 2009), thus demonstrating the ability of virus to involve either the central or peripheral nervous system. The present report illustrates a case of PB19 infection characterized by cranial polyneuropathy and hemolytic

Table 1 Causes of multiple cranial neuropathies involving the second and third cranial nerves

\begin{tabular}{llll}
\hline Infectious & Inflammatory & Vascular & Neoplastic \\
\hline Viruses: & Sarcoidosis & Carotid cavernous fistula & Head and neck tumors \\
Epstein-Barr virus & Systemic lupus erythematosus & Carotid cavernous aneurysm & Neural tumors \\
Human immunodeficiency virus & Churg-Strauss syndrome & Cavernous sinus thrombosis & Hematologic malignancies \\
Varicella zoster virus & Wegener granulomatosis & Sickle cell anemia & Metastatic lesions \\
Cytomegalovirus & Giant cell arteritis & & Leptomeningeal carcinomatosis \\
Bacteria: & Tolosa-Hunt syndrome & & \\
Borrelia burgdorferi & & & \\
Treponema pallidum & & & \\
Mycobacterium tuberculosis & & & \\
Fungi: & & & \\
Aspergillosis & & & \\
Mucormycosis & & & \\
Parasites: & & & \\
Toxoplasma gondii & & & \\
\hline
\end{tabular}


anemia in an immunologically healthy woman. Interestingly, no other typical PB19-related symptoms such as rash or arthropathy were observed. Visual loss and ophthalmoplegia are often caused by infectious, inflammatory, neoplastic, and vascular processes (Table 1) (Keane 2005). Detailed microbiological and virological tests ruled out a variety of acute infections, but showed elevated titers of PB19 IgM and IgG. Other inflammatory, neoplastic, or vascular disorders were also excluded based on normal imaging and autoimmune studies. The diagnosis of acute PB19 infection was established with detection of positive DNA and anti-PB19 IgM antibodies in blood samples. As PB19 DNA may be detectable for extended periods of time in serum (Musiani et al. 1995), its detection does not indicate acute infection. However, in our case, the presence of immunoglobulin $\mathrm{M}$ during the acute neurological deficit, together with the simultaneous development of self-limited hemolytic anemia, suggests a pathogenic role of PB19. Although not completely understood, the pathogenesis of PB19 is complex and several mechanisms may be involved during a single infection, including direct effects of the viral particle, dysregulated immune response with cytokine up-regulation, immune complex deposition, and cytotoxic properties of NS1 protein (Barah et al. 2003). In addition, PB19 infection has been shown to be a trigger for development of a vast array of autoimmune conditions and autoantibody production (Kerr 2016). In our case, the transient detection of antiganglioside GD1b antibodies might represent the pathogenic mechanism by which PB19 affected the central and peripheral nervous system. Antiganglioside antibodies were previously described in PB19-associated Guillain-Barré syndrome (Yamaoka et al. 2000) but not in PB19-related central nervous system manifestations. In our view, PB19 infection induced serum antibody production against GD1b by means of an eventual molecular mimicry mechanism (Kerr 2016). The concept of PB19induced immune dysfunction led to treatment of its neurological complications with corticosteroids and IGIV, though numerous patients improve spontaneously (Qiu et al. 2017). Our patient was primarily treated with IVIG and later with methylprednisolone due to incomplete recovery. Fortunately, subsequent resolution of symptoms was observed 3 months after disease onset despite the persistence of PB19 DNA and anti-PB19 IgM antibodies 5 months after the initial presentation. In conclusion, our report provides evidence that PB19 could affect both the central and peripheral nervous system, possibly by triggering an autoimmune mechanism that leads to autoantibody production. Furthermore, our case also suggests that investigation of PB19 infection should be considered even in the absence of typical associated features and regardless of immune status.

\section{References}

Barah F, Vallely PJ, Cleator GM, Kerr JR (2003) Neurological manifestations of human parvovirus B19 infection. Rev Med Virol 13:185-199

Barah F, Whiteside S, Batista S, Morris J (2014) Neurological aspects of human parvovirus B19 infection: a systematic review. Rev Med Virol 24:154-168

Broliden K, Tolfvenstam T, Norbeck O (2006) Clinical aspects of parvovirus B19 infection. J Intern Med 260:285-304

Douvoyiannis M, Litman N, Goldman DL (2009) Neurologic manifestations associated with parvovirus B19 infection. Clin Infect Dis 48: $1713-1723$

Keane JR (2005) Multiple cranial nerve palsies: analysis of 979 cases. Arch Neurol 62:1714-1717

Kerr JR (2016) The role of parvovirus B19 in the pathogenesis of autoimmunity and autoimmune disease. J Clin Pathol 69:279-291

Musiani M, Zerbini M, Gentilomi G, Plazzi M, Gallinella G, Venturoli S (1995) Parvovirus B19 clearance from peripheral blood after acute infection. J Infect Dis 172:1360-1363

Qiu J, Söderlund-Venermo M, Young NS (2017) Human Parvoviruses. Clin Microbiol Rev 30:43-113

Yamaoka Y, Isozaki E, Kagamihara Y, Matsubara S, Hirai S, Takagi K (2000) A case of Guillain-Barré syndrome (GBS) following human parvovirus B19 infection. Rinsho Shinkeigaku 40:471-475 